\title{
INVARIANT SUBSPACES OF HARDY CLASSES ON INFINITELY CONNECTED PLANE DOMAINS ${ }^{1}$
}

\author{
BY CHARLES W. NEVILLE \\ Communicated by Irving Glicksberg, February 14, 1972
}

Let $\boldsymbol{C}$ be the complex plane, $\boldsymbol{C e}$ the extended plane, $\Delta(a, r)$ the open disk of radius $r$ centered at $a, R$ a Riemann surface and $H^{p}(R)$ Hardy class $H^{p}$ of $R$ (cf. [5, pp. 9-12]). A now classical theorem of Beurling states that the closed subspaces of $H^{2}(\Delta(0,1))$ invariant under multiplication by $z$ are exactly the subspaces $V$ of the form $V=I \cdot H^{2}(\Delta(0,1))$, where $I$ is an inner function determined up to multiplication by a constant of modulus 1 by $V[1]$. Analogous theorems hold for $H^{p}(R)$, where $R$ is the interior of a compact bordered Riemann surface and $1 \leqq p \leqq \infty$. (If $p=\infty$, the proper topology for $V$ to be closed in is either the $\beta$ or bounded weak-star topology of Buck [2], or else the weak-star topology.) (Cf. $[3],[4],[10],[13]$.) We have generalized these theorems to $H^{p}(R)$, where $R$ is a certain type of infinitely connected plane domain.

Before stating our generalization, we must make several definitions. A locally analytic modulus, or l.a.m., is a real valued function $g$ on $R$ such that for each simply connected open subset $U$ of $R$, there exists $f$ analytic on $U$ such that $g=|f|$. The 1.a.m. $g$ is inner if $\log g=G+S$, where $G$ is a sum of Green's functions and $S$ is a singular harmonic function in the sense of Parreau ([8], cf. also [5, p. 7]). If $R=\Delta(0,1)$, an analytic function $I$ is inner in the usual sense [6, pp. 61-68] if and only if the l.a.m. $|I|$ is inner.

$R$ is a Blaschke region in case $R \subseteq C$ and $R$ is of the form $C e$ $\bigcup\{A(i): 0 \leqq i<\infty\}$ (or, $C e \sim \bigcup A(i): 0 \leqq i \leqq n\}$ ) where the $A(i)$ are pairwise disjoint continua such that $C e \sim A(i)$ is connected for each $i$. In addition, there must exist an integer $n$ such that the $A(i)$ cluster only on $\bigcup\{A(i): 0 \leqq i \leqq n\}$, and a sequence $a(i) \in A(i), i \geqq n+1$, such that $\sum(G(a(i), z): n+1 \leqq i<\infty)<\infty$. Here $G(a, z)$ is the Green's function for $C e \sim \bigcup\{A(i): 0 \leqq i \leqq n\}$. Voichick first studied this class of plane regions [13]. We call them Blaschke regions because the prototype of

AMS 1970 subject classifications. Primary 46J15, 30A98; Secondary 30A86, 46J20.

Key words and phrases. Beurling's theorem, Blaschke regions, bounded weak-star topology, Cauchy integral formula, exterior function, Hardy class $H^{p}$, inner function, interior function, infinitely connected plane domains, invariant subspaces, modules and rings of analytic functions, Read's theorem, shift operators.

${ }^{1}$ These results comprise part of my doctoral dissertation at the University of Illinois, 1972. I thank my advisor, L. A. Rubel, for his generous advice and patience. Also, portions of this work were done while the author was an Instructor at Washington University, St. Louis, Missouri 63130. 
such a region is a region $R$ of the form $R=\Delta(0,1) \sim \bigcup A(i): 1 \leqq i<\infty\}$; where the $A(i)$ 's are as above for $1 \leqq i<\infty$, cluster only on $\partial \Delta(0,1)$, and such that there exists a convergent Blaschke product with zeroes $a(i) \in A(i), 1 \leqq i<\infty$.

Let $R=\boldsymbol{C} e \sim \bigcup A(i): 0 \leqq i<\infty\}$ be a Blaschke region. Since each $C e \sim A(i)$ is simply connected and each $A(i)$ is a continuum, we may map each $C e \sim A(i)$ into $\Delta(0,1)$ via the Riemann mapping function $\psi(i)$. For $n+1<i<\infty$, let $\Gamma(i)=\partial \Delta(0,1) \times\{A(i)\}$, and for $0 \leqq i \leqq n$, let $\Gamma(i)=(\partial \Delta(0,1) \sim E(i)) \times\{A(i)\}$, where $E(i)$ is the set of cluster points of the $\psi(i)(A(j)), j \neq i$. Let $\Gamma=\bigcup\{\Gamma(i): 0 \leqq i<\infty\}$. We endow $R \cup \Gamma$ with the appropriate topology and conformal structure, which agrees on $R$ with the ones inherited from $C . R \cup \Gamma$ is then a bordered Riemann surface. $\Gamma$ is called the canonical border of $R$. We now may state the main theorem.

TheOREM A. Let $R$ be a Blaschke region, $\Gamma$ the canonical border of $R$, and suppose the ideal boundary of $R \cup \Gamma$ has harmonic measure 0 . Then

(i) Each $\beta$ closed ideal of $H^{\infty}(R)$ is of the form

$$
\left\{f \in H^{\infty}(R):|f| / I \text { is bounded }\right\}
$$

for a unique bounded inner l.a.m. I. Conversely, each set of the above form is a $\beta$ closed ideal.

(ii) Let $1 \leqq p<\infty$. Each norm closed $H^{\infty}(R)$ submodule of $H^{p}(R)$ is of the form

$$
\left\{f \in H^{p}(R):(|f| / I)^{p} \text { has a harmonic majorant }\right\}
$$

for a unique bounded inner l.a.m. I. Conversely, each set of the above form is a norm closed $H^{\infty}(R)$ submodule.

Our proof of Theorem A is modeled on Rudin's proof of the BeurlingRudin characterization of the closed ideals of the algebra of functions analytic on $\Delta(0,1)$ and continuous on $C 1(\Delta(0,1))$ (cf. [6, pp. 85-87]). In many respects our proof also parallels Voichick's proof of the analogue of Theorem A(ii) for compact bordered Riemann surfaces [13].

Our proof utilizes three results of independent interest. The first of these, Theorem B, was obtained independently by $\mathrm{H}$. Widom, who gave a proof more elegant than ours [15]. Throughout, $\delta U=d U+i * d U$, $R$ is a Blaschke region, $b$ a fixed point in $R, G(a, z)$ the Green's function for $R$, and $\mathscr{Z}$ the set of zeroes of $\delta G(b, z)$ counting multiplicity.

THEOREM B. $\sum(G(z, w): z \in \mathscr{Z})<\infty$ for each $w \in R \sim \mathscr{Z}$.

In Theorems $\mathrm{C}$ and $\mathrm{D}, R \cup \Gamma$ satisfies the hypotheses of Theorem $\mathrm{A}$. Further, $g(w)=\exp \left(-\sum(G(z, w): z \in \mathscr{Z})\right)$. The function $g$ is, of course, 
an inner bounded 1.a.m. Finally, if $f$ is an extended complex valued function defined on $R$, we shall denote the nontangential (sectoral) limit of $f$ at $p$ by $f^{*}(p)$ for each $p \in \Gamma$ where the limit is defined.

THEOREM C. Let $f$ be meromorphic on $R$ and suppose $|f| g$ has a harmonic majorant. Then $f^{*}$ exists a.e. on $\Gamma$ and is integrable with respect to harmonic measure. Further,

$$
f(b)=-\frac{1}{2 \pi i} \int_{\Gamma} f^{*}(z) \delta G(b, z),
$$

where $\Gamma$ is oriented positively with respect to $R$.

THEOREM D. Let $u$ be integrable on $\Gamma$ with respect to harmonic measure. Suppose

$$
\int_{\Gamma} h^{*}(z) u(z) \delta G(b, z)=0
$$

for each function $h$, meromorphic on $R$, such that $g|h|$ is bounded and $h(b)=0$. Then there exists a function $f \in H^{1}(R)$ such that $f^{*}=u$ a.e. on $\Gamma$ with respect to harmonic measure.

It is readily verified that Theorem $\mathrm{C}$ is a form of the Cauchy Integral Formula $(f(z) \delta G(b, z)$ has residue -1 at $z=b)$ and Theorem $\mathrm{D}$ is a version of Read's theorem [9]. (We are indebted to J. A. Jenkins for the observation that Read's theorem for $\mathrm{Cl}(\Delta(0,1))$ is a consequence of Morera's theorem. Our proof of Theorem D is an extension of his observation.) The reader should also note that harmonic measure on $\Gamma$ at $b$ is simply given by $-(1 / 2 \pi i) \delta G(b, z)$.

We also have a counterexample, based on Rudin type bubble regions [12], showing that Theorem A(i) does not hold for all infinitely connected plane regions $R$, even if $R$ admits enough bounded analytic functions to separate points. C. W. Kennel has recently generalized our example considerably [7].

Finally, we have a counterexample showing that not every closed submodule of $H^{p}(R)$ is of the form $\psi \cdot H^{p}(R)$ for some $\psi \in H^{\infty}(R)$ even if $R$ is a Blaschke region satisfying the hypotheses of Theorem A. This construction gives an example of a function $\psi \in H^{\infty}(R)$ which has no interior-exterior factorization in the sense of Rubel-Shields ([10], [11]).

Details will appear elsewhere.

\section{REFERENCES}

1. A. Beurling, On two problems concerning linear transformations in Hilbert space, Acta Math. 81 (1948), 17 pp. MR 10, 381. 
2. R. Buck, Algebraic properties of classes of analytic functions, Seminars on Analytic Functions, vol. 2, Princeton, N.J., 1957, 175-188.

3. M. Hasumi, Invariant subspace theorems for finite Riemann surfaces, Canad. J. Math. 18 (1966), 240-255. MR 32 \#8200.

4. M. Hasumi and T. Srinivasan, Doubly invariant subspaces. II, Pacific J. Math. 14 (1964), 525-535. MR 29 \#1529.

5. M. Heins, Hardy classes on Riemann surfaces, Lecture Notes in Math., no. 98, Springer-Verlag, Berlin and New York, 1969. MR 40 \#338.

6. K. Hoffman, Banach spaces ofanalytic functions, Prentice-Hall Series in Modern Analysis, Prentice-Hall, Englewood Cliffs, N.J., 1962. MR 24 \# A2844.

7. C. Kennel, Locally outer functions (to appear).

8. M. Parreau, Sur les moyennes des fonctions harmoniques et analytiques et la classification des surfaces de Riemann, Ann. Inst. Fourier (Grenoble) 3 (1951), 103-197. MR 14, 263.

9. A. H. Read, A converse of Cauchy's theorem and applications to extremal problems, Acta Math. 100 (1958), 1-22. MR 20 \#4640.

10. L. A. Rubel and A. L. Shields, The space of bounded analytic functions on a region, Ann. Inst. Fourier (Grenoble) 16 (1966), fasc. 1, 235-277. MR 33 \#6440.

11. - The failure of interior-exterior factorization, Tôhoku Math. J. (to appear).

12. W. Rudin, Essential boundary points, Bull. Amer. Math. Soc. 70 (1964), 321-324. MR 28 \#3167.

13. M. Voichick, Ideals and invariant subspaces of analytic functions, Trans. Amer. Math. Soc. 111 (1964), 493-512. MR 28 \#4129.

14. - Extreme points of bounded analytic functions on infinitely connected regions, Proc. Amer. Math. Soc. 17 (1966), 1366-1369. MR 33 \#7881.

15. H. Widom, The maximum principle for multiple-valued analytic functions, Acta Math. 126 (1971), 63-82. MR 43 \#5034.

Department of Mathematics, University of Texas at El Paso, El Paso, Texas 79968

Current address: DePartment of Mathematics, University of NeW Hampshire, DURHAM, NEW HAMPSHIRE 03824 